\title{
Efectos del bypass gástrico en el esófago de Barrett de pacientes con obesidad mórbida*
}

\author{
Drs. NICOLÁS PEREIRA C. ${ }^{1}$, ATTILA CSENDES J. ${ }^{1}$, GLADYS SMOK S. ${ }^{2}$, \\ ANA MARÍA BURGOS L. ${ }^{1}$, SOLANGE NÚÑEZ B. ${ }^{1}$ \\ 1 Departamento de Cirugía. Hospital Clínico Universidad de Chile. \\ 2 Departamento de Anatomía Patológica. Hospital Clínico Universidad de Chile. \\ Santiago, Chile.
}

\begin{abstract}
Effects of gastric bypass for morbid obesity on Barrett esophagus

Background: Besides the weight reducing effects of gastric bypass, it is also a good antireflux procedure since there is no acid production by the gastric pouch and there is no duodenal reflux due to the presence of a Roux en Y. Aim: To describe the effect of gastric bypass on Barrett esophagus among patients with morbid obesity. Material and Methods: Among 896 patients subjected to gastric bypass, 14 patient with a Barrett esophagus diagnosed with endoscopy and biopsy, were followed. A new endoscopy was performed one to 30 months after the surgical procedure. Results: Short $(<=30 \mathrm{~mm})$ and long segment $(>=31 \mathrm{~mm})$ Barrett esophagi were present in eight and six patients, respectively. Gastroesophageal reflux symptoms relieved in $70 \%$ of these cases in a mean lapse of 6.5 months. There was regression from intestinal metaplasia to cardial mucosa in six patients (75\%) with short-segment, and in one patient (16\%) with long-segment Barrett esophagus. Conclusions: Gastric bypass in patients with morbid obesity and Barrett esophagus is a very good antireflux operation. This was proved by the disappearance of symptoms in almost all patients and by the regression of the intestinal metaplasia which is time and length dependent.
\end{abstract}

Key words: Gastric bypass, Barrett esophagus, morbid obesity.

\section{Resumen}

Antecedentes: El bypass gástrico en obesos mórbidos, además de ser una alternativa de cirugía bariátrica, es un buen procedimiento antirreflujo dado que no hay producción de ácido en el reservorio gástrico y no existe reflujo duodenal debido a la Y de Roux. Objetivo: Describir el efecto que tiene el bypass gástrico sobre el esófago de Barrett (EB) de pacientes obesos mórbidos, además discutir nuevos mecanismos fisiopatológicos implicados. Población: De 896 obesos mórbidos operados mediante bypass gástrico resectivo abierto, se siguieron a los 14 pacientes diagnosticados con EB mediante endoscopia e histología. Resultados: De los 14 pacientes con diagnóstico de esófago de Barrett, ocho pacientes presentaban EB corto $(\leq 30 \mathrm{~mm})$ y seis EB largo ( $\geq 31 \mathrm{~mm}$ ). Los síntomas de pirosis y/o regurgitación presentes se resolvieron en un $70 \%$ de los casos en una media de 6,5 meses. Hubo una regresión de la metaplasia intestinal a mucosa cardial normal en seis pacientes $(75 \%)$ con EB corto y en un paciente (16\%) con EB largo. Conclusiones: El bypass gástrico es una muy buena alternativa quirúrgica antirreflujo en obesos mórbidos con EB, demostrado por la desaparición

*Recibido el 12 de julio de 2011 y aceptado para publicación el 3 de septiembre de 2011.

Correspondencia: Dr. Nicolás Pereira C.

Santos Dumont 999. Santiago, Chile.

nicolaspereirac@gmail.com 
de los síntomas en la mayoría de los pacientes y la regresión de la metaplasia intestinal en la mitad de ellos, dependiendo del tiempo y de la longitud. Lo anterior, postulamos, que no sería debido solamente a que no hay producción de ácido en el reservorio gástrico y a que no existe reflujo duodenal, sino que habrían otros mecanismos fisiopatológicos implicados.

Palabras clave: Obesidad mórbida, esófago de Barrett, bypass gástrico, metaplasia intestinal.

\section{Introducción}

En 1950, Nunan Barrett, describió úlceras pépticas crónicas en el esófago rodeadas de epitelio columnar ${ }^{1}$. Veinte años más tarde, Trier descubrió que el epitelio en el esófago de Barrett recordaba al de tipo intestinal ${ }^{2}$. El esófago de Barrett (EB) es el cambio metaplásico de la mucosa del esófago, de manera que el epitelio escamoso normal es reemplazado por un epitelio columnar intestinal ${ }^{3}$. Este trastorno se puede presentar como una complicación del reflujo gastroesofágico (RGE), aunque individuos asintomáticos pueden estar afectados de igual manera. Por otro lado, la obesidad constituye un estado proinflamatorio en el cual además existe mayor resistencia a la insulina, lo cual contribuiría en la patogénesis de esta enfermedad ${ }^{4,5}$. Constituye un factor de riesgo para el desarrollo del adenocarcinoma esofágico ${ }^{6}$, cuya incidencia ha aumentado rápidamente en sociedades desarrolladas. Un estudio sueco mostró una prevalencia global de 1,6\%, de los cuales un tercio correspondía a segmento $\operatorname{largo}{ }^{7}$ ( $\geq 31 \mathrm{~mm}$ ), mientras que en un estudio americano con pacientes sanos que se sometieron a endoscopia digestiva alta y colonoscopia para detección de cáncer colorrectal la prevalencia fue $5,6 \%{ }^{8}$.

En pacientes obesos mórbidos, el bypass gástrico es la "cirugía de elección",10". Disminuye notablemente los síntomas de reflujo gastroesofágico, así como la cantidad de ácido en el reservorio gástrico ${ }^{11}$. Además el asa en Y de Roux desvía la bilis y el contenido duodenal, evitando el reflujo al estómago y esófago distal. Debido a esto, pareciera ser la operación ideal en el tratamiento del reflujo crónico en pacientes obesos mórbidos con esófago de Barrett, sin embargo, su efectividad sobre la presencia, longitud de éste y su efecto en el tiempo no está bien definida.

El objetivo del presente estudio prospectivo fue describir los efectos en el tiempo que tiene el bypass gástrico resectivo sobre el EB de pacientes obesos mórbidos operados y discutir nuevos mecanismos fisiopatológicos que estarían implicados en estos efectos.

\section{Material y Método}

\section{Pacientes estudiados}

Este estudio realizó un seguimiento en el tiempo de los pacientes con diagnóstico de EB en la evalua- ción preoperatoria, que incluía endoscopia digestiva alta y biopsia. Se consideró a los 896 pacientes con obesidad mórbida sometidos a bypass gástrico abierto resectivo entre los años 1998 y 2009, de los cuales 14 presentaron biopsia compatible con EB $(1,56 \%)$.

\section{Examen endoscópico}

Uno de los autores (A.C.), realizó todos los procedimientos endoscópicos, usando un endoscopio Olympus GYFXQ-20. El procedimiento se llevó a cabo después de 12 horas de ayuno. Se usó lidocaína como anestésico local en faringe. El epitelio columnar esofágico distal, fue medido como la distancia entre la unión escamo-columnar y la localización endoscópica ubicada bajo el esfínter esofágico distal, que es el punto de unión del epitelio columnar del esófago y los pliegues gástricos. Se registraron todas las lesiones ulcerativas de la unión escamo-columnar. Las endoscopias digestivas, se hicieron previo a la cirugía y posterior a ella, tomando muestras para biopsias de 4 cuadrantes, a unos $5 \mathrm{~mm}$ distal de la unión escamo-columnar. Se tomaron entre 6-16 muestras para biopsia en cada paciente.

\section{Regresión del EB}

Se definió regresión como: a) A la desaparición de la metaplasia intestinal en las biopsias realizadas en los controles postoperatorios; b) A la disminución de al menos $3 \mathrm{~cm}$ de longitud del epitelio columnar.

\section{Análisis histológico}

Todas las muestras fueron sumergidas de inmediato en solución de formalina al 10\% y enviadas a evaluación histológica, después de teñir con hematoxicilina-eosina y azul alcian a un $\mathrm{pH}$ 2,5. La mucosa fúndica fue identificada por la presencia de células parietales en la profundidad de las capas glandulares y la mucosa cardial fue identificada por la presencia de secreción mucosa por las células columnares. La metaplasia intestinal se definió con la presencia de células caliciformes. También se buscó la presencia de $H$. pylori en la mucosa columnar con metaplasia intestinal.

\section{Procedimiento quirúrgico}

En todos los pacientes se realizó un bypass gástrico abierto resectivo, dejando un reservorio gástrico pequeño, con menos de $20 \mathrm{ml}$ de capacidad y cons- 
truido con stapler (Tyco Healthcare, Norwalk, CT). La gastro-yeyunoanastomosis se hizo con stapler circular 25 (Tyco Healthcare). La longitud del asa alimentaria de la Y de Roux varió entre 125 y $150 \mathrm{~cm}$.

\section{Análisis estadístico}

Se realizó el test de comparación de dos proporciones ( $t$ test) para comparar los cambios en la regresión del $\mathrm{EB}$, un test de dócima de diferencias de medias pareadas para el IMC y el test de McNemar para analizar el cambio en los síntomas y la presencia de H. pylori. Se utilizó programa computacional STATA 9.2. Se consideró como significativo un $\mathrm{p}<0,05$.

\section{Seguimiento}

Todos los pacientes con metaplasia intestinal fueron seguidos y controlados, realizándose la primera endoscopia digestiva alta entre 1 y 30 meses después de la cirugía, manteniendo controles de algunos en el tiempo hasta por 123 meses posterior a ésta.

\section{Resultados}

Después de la cirugía la mayoría de los pacientes experimentó una mejoría. Se realizó un seguimiento de los 14 pacientes con diagnóstico de EB sometidos a by-pass gástrico, de los cuales $35,71 \%$ corresponden a hombres y $64,28 \%$ a mujeres, con un promedio de edad de 47,7 años y rangos que varían entre 33 y 62 años.

La Tabla 1 muestra los hallazgos endoscópicos e histológicos antes y después de la cirugía. La media de seguimiento de los pacientes incluidos en este estudio fue 60 meses.

El grupo de pacientes se dividió en dos; EB corto $(\leq 30 \mathrm{~mm})$ con ocho pacientes y seis pacientes con EB largo $(\geq 31 \mathrm{~mm})$. El porcentaje de regresión de la metaplasia intestinal fue de $75 \%(p=0,0154)$ y $16 \%(\mathrm{p}>0,05)$ respectivamente para ambos grupos. El tiempo promedio de regresión de EB corto fue 28 meses. Un 12,5\% tuvo una regresión en 12 meses, mientras que un $50 \%$ ya había regresado a los 36 meses. Continuando con el control de estos pacientes se evidenció que a los 48 meses un $62,5 \%$ ya no presentaba metaplasia intestinal en la biopsia endoscópica.

Se aprecia además un porcentaje significativo de reducción de índice de masa corporal (IMC), siendo el promedio preoperatorio de $41,7 \mathrm{~kg} / \mathrm{mt}^{2}$ (rango 33 a 61) y postoperatorio de $28,8 \mathrm{~kg} / \mathrm{mt}^{2}$ (rango 21,7 a 42,8 ) a los 24 meses de control, lo que corresponde a una disminución promedio de un $31,5 \%$ en 24 meses $(p=0,0000)$. Se evaluó también los síntomas de RGE presentes en el preoperatorio (epigastralgia, pirosis, regurgitación) de 10 de los pacientes los que se resolvieron en un $70 \%$ en el postoperatorio en un período de tiempo promedio de 6,5 meses $(\mathrm{p}=0,0156)$. De los 14 pacientes, seis presentaron $(42,85 \%) H$. pylori (+) en el estudio preoperatorio, el cual desapareció posteriormente en el $66,6 \%$, sin mediar tratamiento específico $(\mathrm{p}=0,1797)$.

\section{Discusión}

Los resultados del presente estudio sugieren la utilidad del bypass gástrico como cirugía antirreflujo en pacientes obesos mórbidos con $\mathrm{EB}$, demostrado por la regresión de la metaplasia intestinal en un gran porcentaje de los enfermos $(75 \%$ en EB corto y $16 \%$ en EB largo).

Si bien hay una relación directa entre el incremento del IMC y la sintomatología del RGE, no existe tal asociación con la presencia de EB. Sin embargo, la circunferencia abdominal constituye un factor de riesgo independiente para el desarrollo de esta patología ${ }^{4}$.

Algunos estudios establecen el efecto que tiene el bypass gástrico en disminuir o eliminar los síntomas de RGE ${ }^{12}$. El efecto del RGE es un factor importante en el desarrollo del EB. La exposición del esófago distal al reflujo ácido del estómago está usualmente asociado a EB, pero el reflujo de biliar es más común en esta enfermedad ${ }^{13}$. Aunque la bilis por sí sola pareciera no contribuir, existiría un efecto sinérgico entre el ácido y la bilis en la aparición de metaplasia intestinal ${ }^{14,15}$. No existe evidencia que establezca una relación entre la infección por $H$. pylori y el EB, por lo que se requieren más estudios que aclaren esta asociación.

Aunque hay muy pocos estudios respecto al efecto de la cirugía bariátrica, más específicamente, del bypass gástrico en el $\mathrm{EB}$, todos apuntan en el mismo sentido. Cobey, reportó el año 2005 la regresión del EB en una paciente obesa mórbida luego de una cirugía de bypass gástrico ${ }^{16}$. Csendes, en un período de cinco años, siguió 12 pacientes obesos mórbidos con EB operados de bypass gástrico, demostrando una regresión a mucosa cardial normal en el 57\% de los EB de segmento corto y un $20 \%$ en los de segmento largo ${ }^{17}$. Houghton, de la Clínica Mayo, en un estudio retrospectivo de 5 pacientes reportó que todos experimentaron una disminución en la longitud del EB, regresando por completo en 2 pacientes y mejorando el grado de displasia en 3 de ellos ${ }^{18}$.

Los efectos fisiopatológicos que tiene el bypass gástrico, que contribuirían a la regresión del EB, son que la secreción ácida es reducida a un mínimo por una resección de las células parietales con la creación del reservorio gástrico, y la Y de Roux de 125 a $150 \mathrm{~cm}$ produce una abolición permanente $\mathrm{y}$ completa del reflujo duodenal. 


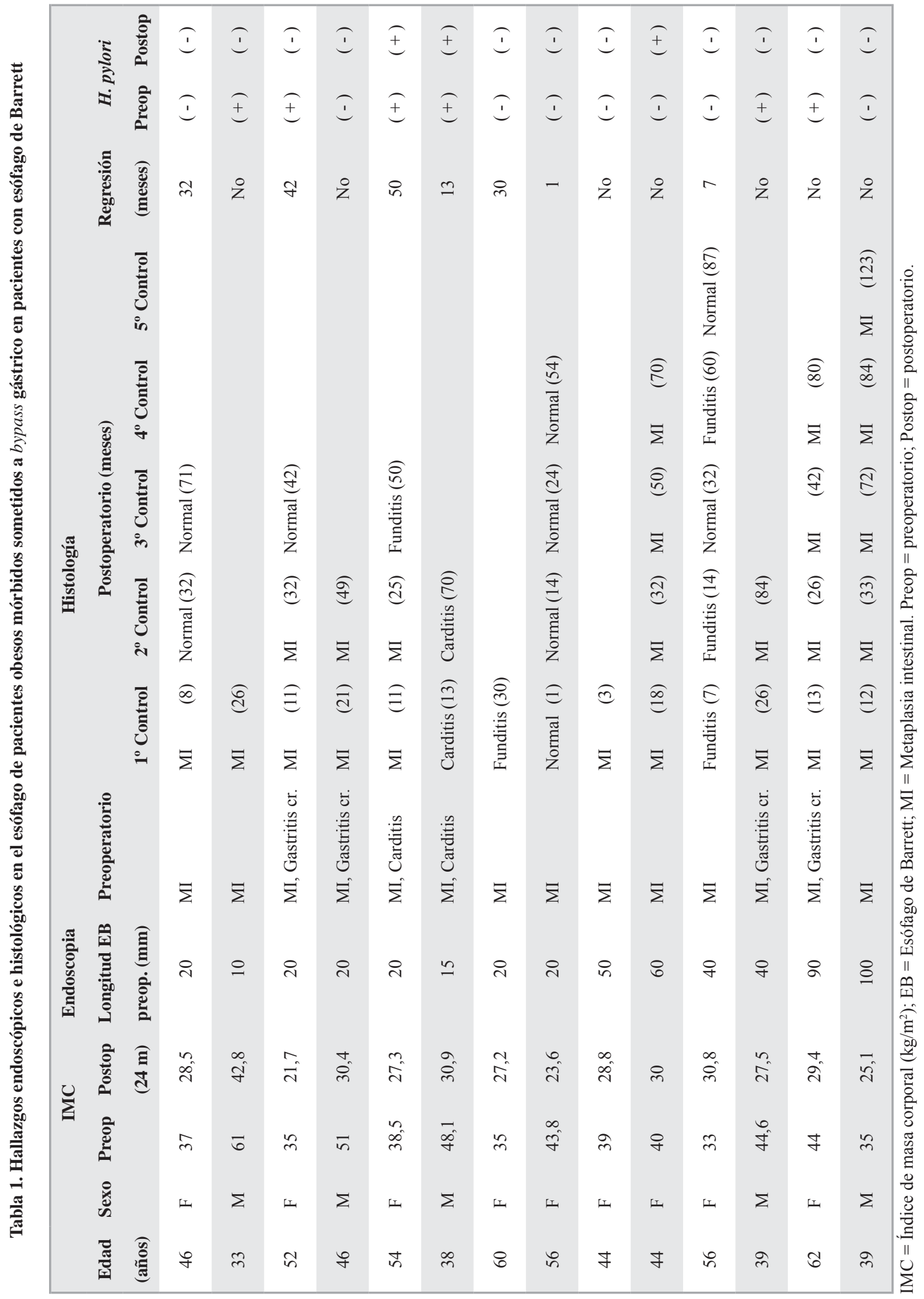




\section{Descripción de nuevos mecanismos fisiopatológicos}

La cirugía antirreflujo en EB también logra la regresión de la metaplasia intestinal, pero no en las proporciones a las obtenidas por nuestro estudio ${ }^{19,20}$. Otros mecanismos fisiopatológicos que explicarían nuestros resultados y las diferencias con la cirugía antirreflujo, es que el bypass gástrico es una técnica significativa para la reducción de peso. La grasa visceral está fuertemente asociada con el incremento en la liberación de citoquinas proinflamatorias y con menores niveles de adiponectina, la cual tiene un efecto antiapoptótico y antiproliferativo, por lo que en conjunto aumenta la inflamación y por ende, la transformación maligna en pacientes con $\mathrm{RGE}^{4}$. Por otro lado, los pacientes con obesidad central tienen mayores concentraciones de insulina e IGF-1, lo cual promueve la proliferación y determina mayor diferenciación celular que contribuiría al desarrollo de metaplasia intestinal ${ }^{5}$. El bypass gástrico reduce la resistencia a la insulina, lo que sumado a la disminución en el peso, más específicamente del diámetro abdominal y la grasa visceral, contribuiría a la regresión del EB.

\section{Conclusiones}

Este trabajo puede presentar error de muestreo, al igual que en cualquier estudio de estas características. Aunque se trató de obtener al menos 8 biopsias durante el procedimiento endoscópico, la posibilidad de error no puede ser descartada. Esta investigación representa la continuación del seguimiento de Csendes, en donde además de ampliar la muestra incorporando nuevos casos, se discuten nuevos mecanismos fisiopatológicos implicados en la resolución del EB. Aquí se reafirma lo planteado anteriormente y se obtienen mejores resultados, con una regresión de la metaplasia intestinal en el $75 \%$ de los pacientes con EB corto en una media de 28 meses, $\mathrm{y}$ en el $16 \%$ con EB largo. Todo lo anterior pone en evidencia que el comportamiento del EB luego del bypass gástrico en pacientes obesos mórbidos, tiende a la regresión y a que no exista progresión de la displasia, lo cual es dependiente de la longitud (inversamente relacionado con la longitud del EB) y dependiente del tiempo (directamente relacionado con el tiempo de seguimiento). Por todo lo expuesto, proponemos que el bypass gástrico debe ser la técnica de elección en pacientes obesos mórbidos con reflujo gastroesofágico patológico y/o esófago de Barrett, dado la fisiopatología y los efectos que tiene este procedimiento en el comportamiento de la metaplasia intestinal.

\section{Referencias}

1. Barrett NR. Chronic peptic ulcer of the oesophagus and "oesophagitis". Br J Surg. 1950-1951;38:175-82.

2. Trier JS. Morphology of the epithelium of the distal esophagus in patients with midesophageal peptic strictures. Gastroenterology 1970;58:444-61.

3. Odze RD. Barrett esophagus: histology and pathology for the clinician. Nat Rev Gastroenterol Hepatol. 2009;6:478-90.

4. El-Serag H. The Association Between Obesity and GERD: A Review of the Epidemiological Evidence. Dig Dis Sci. 2008 53:2307-12.

5. Shaheen N, Richter J. Barrett's oesophagus. Lancet 2009; 373:850-61.

6. Shaheen N, Ransohoff DF. Gastroesophageal Reflux, Barrett Esophagus, and Esophageal Cancer. JAMA 2002;287:1972-81.

7. Ronkainen J, Aro P, Storskrubb T, Johansson SE, Lind T, Bolling-Sternevald E, et al. Prevalence of Barrett's esophagus in the general population: an endoscopic study. Gastroenterology 2005;129:1825-31.

8. Rex DK, Cummings OW, Shaw M, Cumings MD, Wong RK, Vasudeva RS, et al. Screening for Barrett's esophagus in colonoscopy patients with and without heartburn. Gastroenterology 2003;125:1670-77.

9. Brolin RE. Gastric bypass. Surg Clin N Am. 2001;81: 1077-85.

10. Fobi MAL, Lee H, Holness L, Cabinda DG. Gastric bypass operation for obesity. World J Surg. 1998;22: 925-35.

11. Nelson LG, González R, Haines K, Gallagher SF, Murr MM. Amelioration of gastroesophageal reflux symptoms following Roux-en-Y gastric bypass for clinically significant obesity. Am Surg. 2005;71:950-4.

12. Perry Y, Courcoulas AP, Fernando HC, Buenaventura PO, McCaughan JS, Luketich JD, et al. Laparoscopic Roux-en-Y Gastric Bypass for Recalcitrant Gastroesophageal Reflux Disease in Morbidly Obese Patients. JSLS 2004;8:19-23.

13. Vaezi MF, Richter JE. Role of acid and duodenogastroesophageal reflux in gastroesophageal refl ux disease. Gastroenterology 1996;111:1192-9.

14. Avidan B, Sonnenberg A, Schnell TG, Sontag SJ. Gastric surgery is not a risk for Barrett's esophagus or esophageal adenocarcinoma. Gastroenterology 2001;121:1281-85.

15. Dvorak K, Payne CM, Chavarria M, Ramsey L, Dvorakova $\mathrm{B}$, Bernstein $\mathrm{H}$, et al. Bile acids in combination with low $\mathrm{pH}$ induce oxidative stress and oxidative DNA damage: relevance to the pathogenesis of Barrett's oesophagus. Gut 2007;56:763-71.

16. Cobey F, Oelschlager B. Complete Regression of Barrett's Esophagus after Roux-en-Y Gastric Bypass. Obes Surg. 2005;15:710-2.

17. Csendes A, Burgos AM, Smok G, Burdiles P, Henriques 
N. PEREIRA C. y cols.

A. Effect of gastric bypass on Barrett's esophagus and intestinal metaplasia of the cardia in patients with morbid obesity. J Gastrointest Surg. 2006;10:259-64.

18. Houghton S, Romero Y, Sarr M. Effect of Roux-en-Y gastric bypass in obese patients with Barrett's esophagus: attempts to eliminate duodenogastric reflux. Surg for Obes and Rel Dis. 2008;4:1-5.
19. Oelschlager BK, Barreca M, Chang L, Oleynikov D, Pellegrini CA. Clinical and Pathologic Response of Barrett's Esophagus to Laparoscopic Antireflux Surgery. Ann Surg. 2003;238:458-66.

20. O'Riordan JM, Byrne PJ, Ravi N. Long-term clinical and pathologic response of Barrett's esophagus after antireflux surgery. The Am J of Surg. 2004;188:27-33. 